\title{
Density functional theory calculations of anisotropic constitutive relationships in alpha-cyclotrimethylenetrinitramine
}

\author{
M. W. Conroy, ${ }^{1, a)}$ I. I. Oleynik, ${ }^{1}$ S. V. Zybin, ${ }^{2}$ and C. T. White ${ }^{3}$ \\ ${ }^{1}$ Department of Physics, University of South Florida, Tampa, Florida 33620, USA \\ ${ }^{2}$ Materials and Process Simulation Center, California Institute of Technology, Pasadena, \\ California 91125, USA \\ ${ }^{3}$ Naval Research Laboratory, Washington, DC 20375, USA
}

(Received 11 August 2008; accepted 9 October 2008; published online 1 December 2008)

Constitutive relationships in the crystalline energetic material $\alpha$-cyclotrimethylenetrinitramine $(\alpha$-RDX) have been investigated using first-principles density functional theory. The equilibrium properties of $\alpha$-RDX including unit cell parameters and bulk modulus, as well as the hydrostatic equation of state (EOS), have been obtained and compared with available experimental data. The isotropic EOS has been extended to include the anisotropic response of $\alpha$-RDX by performing uniaxial compressions normal to several low-index planes, $\{100\},\{010\},\{001\},\{110\},\{101\},\{011\}$, and $\{111\}$, in the Pbca space group. The uniaxial-compression data exhibit a considerable anisotropy in the principal stresses, changes in energy, band gaps, and shear stresses, which might play a role in the anisotropic behavior of $\alpha$-RDX under shock loading. (c) 2008 American Institute of Physics.

[DOI: $10.1063 / 1.3031216]$

\section{INTRODUCTION}

Cyclotrimethylenetrinitramine (1,3,5-trinitrohexahydro1,3,5-triazine), also termed RDX, is a major secondary explosive that is widely used in munitions in cast and plasticbonded formulations. Owing to its importance as an energetic material, RDX has been the subject of extensive experimental ${ }^{1-14}$ and theoretical ${ }^{15-22}$ studies. It is widely accepted that RDX possesses at least three polymorphs, with $\alpha$-RDX as the most stable structure at ambient conditions. ${ }^{1,5}$ The structure of $\alpha$-RDX was studied by Choi and Prince ${ }^{2}$ using X-ray diffraction. They found that the $\alpha$ phase belongs to the orthorhombic space group $\mathrm{Pbca}$ and has eight $\mathrm{C}_{3} \mathrm{H}_{6} \mathrm{~N}_{6} \mathrm{O}_{6}$ molecules (168 atoms) per unit cell. ${ }^{2}$

The hydrostatic equation of state (EOS) of RDX at room temperature was obtained by Olinger et al. ${ }^{3}$ and Yoo et al. ${ }^{4}$ Both experiments ${ }^{3,4}$ found a phase transition from $\alpha$-RDX to $\gamma$-RDX near 4 GPa. Subsequently, Raman spectroscopy has been employed for a detailed investigation of RDX at pressures up to about $4 \mathrm{GPa}$ by Ciezak et al. ${ }^{6}$ and Dreger and Gupta, ${ }^{7}$ who found features in the spectra that are associated with the phase transition at this pressure. Very recently, the structure of the $\gamma$ polymorph has been reported by Davidson et $a l^{8}{ }^{8}$ using a combination of $\mathrm{x}$-ray and neutron diffraction. Few studies have been performed on the high temperature $\beta$ phase because of its structural instability. ${ }^{5,9}$ A theoretical investigation of hydrostatic compression of $\alpha$-RDX was also performed by Byrd and Rice ${ }^{22}$ using density functional theory (DFT).

Elastic properties of $\alpha$-RDX have also been investigated by both experiment and theory. In particular, the bulk modulus has been reported ${ }^{3,4,8}$ by fitting hydrostatic $P-V$ data to various EOS forms. In addition, the elastic constants, bulk

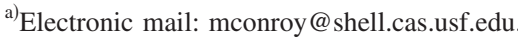

moduli, and shear moduli have been measured experimentally using a sound-propagation technique, ${ }^{10}$ Brillouin scattering, ${ }^{11}$ and resonant ultrasound spectroscopy. ${ }^{12}$ Sewell and Bennett ${ }^{20}$ performed isothermal-isobaric Monte Carlo calculations on RDX from 218 to $333 \mathrm{~K}$ and from 0 to $4 \mathrm{GPa}$ and obtained the elastic constants in good agreement with experimental measurements.

To study the shock sensitivity of RDX, Hooks et al. ${ }^{13}$ performed shock-compression experiments. Shocks were delivered perpendicular to the (111), (210), and (100) planes, each direction having a different number of available slip systems. ${ }^{13}$ The results might indicate that the elastic response of RDX is anisotropic. ${ }^{13}$ Further, there was unexpected behavior of the shock profiles delivered normal to the (100) plane, which Hooks et al. ${ }^{13,14}$ later concluded was due to cracks in the sample.

Shock compression of an energetic material involves uniaxial compression of the crystal. An understanding of the anisotropic response of an energetic material upon uniaxial compression might facilitate an understanding of fundamental mechanisms of shock initiation of detonation in the material. This paper presents the results of first-principles DFT studies of $\alpha$-RDX under both hydrostatic and uniaxial compressions, the latter being applied normal to the $\{100\},\{010\}$, $\{001\},\{110\},\{101\},\{011\}$, and $\{111\}$ planes. Previous investigations of pentaerythritol tetranitrate (PETN) (Ref. 23) and cyclotetramethylenetetranitramine (HMX) (Ref. 24) revealed a substantial anisotropy in the mechanical properties of these materials under uniaxial loading. Therefore, it is expected that RDX will exhibit the same trend. Of particular interest is the behavior of the shear stresses during compression because of their important role in initiating plastic deformations and their possible contribution to the anisotropic shock sensitivity of energetic materials. 


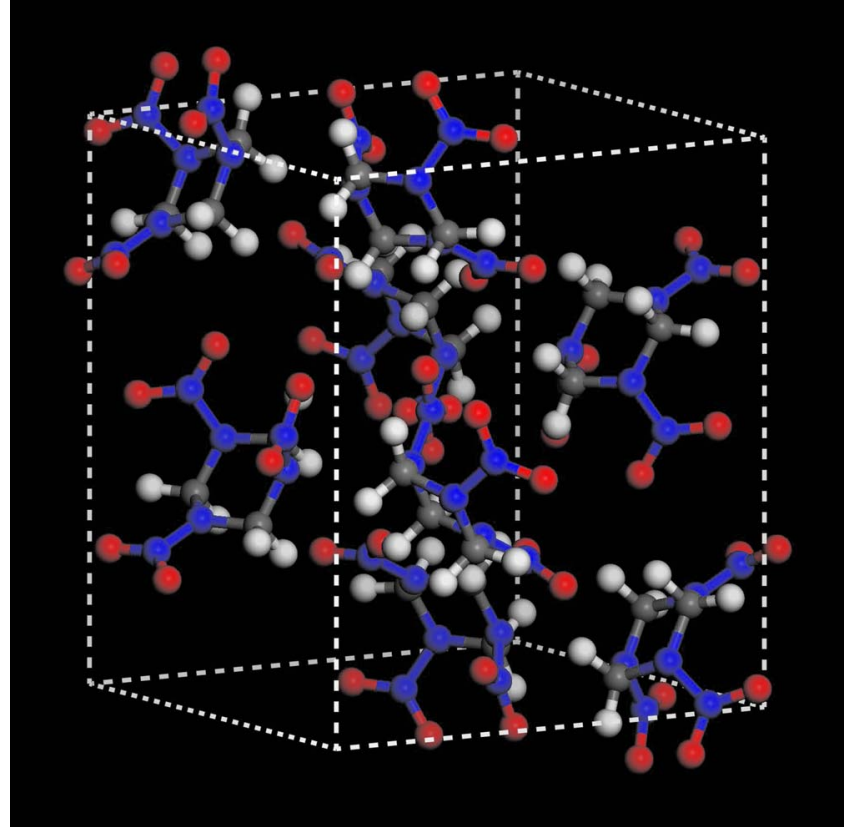

FIG. 1. (Color online) Unit cell of $\alpha$-RDX.

\section{COMPUTATIONAL DETAILS}

We performed first-principles DFT calculations of $\alpha$-RDX using the Vienna ab initio simulation package (VASP) (Refs. 25 and 26). The parameters of the calculations including the exchange-correlation functional, pseudopotential, and energy cutoff were chosen to provide adequate accuracy from a series of tests performed on PETN in our previous work. ${ }^{23}$ Here we used the Perdew-Burke-Ernzerhof (PBE) (Refs. 27 and 28) functional, the projector-augmented wave potentials, ${ }^{29,30}$ and an energy cutoff of $700 \mathrm{eV}$ for all calculations performed on $\alpha$-RDX. Owing to the large size of the unit cell (168 atoms), DFT calculations involving RDX are computationally expensive. This cost was minimized by using only the gamma $k$-point in our calculations.

The experimental structure ${ }^{2}$ of $\alpha$-RDX (space group $P b c a$ ) was relaxed by using the quasi-Newton relaxation algorithm as implemented in VASP to obtain the zero-pressure unit-cell parameters. The structural optimization was performed without any constraints on the unit-cell volume, shape, or symmetry. The tolerance in energy was set to $10^{-6} \mathrm{eV}$ for self-consistent field cycles, and the relaxation was stopped once the maximum force on every atom was less than $0.03 \mathrm{eV} / \AA$.

For the simulation of hydrostatic compression, we scaled the volume of the unit cell from $V / V_{0}=1.00$ to 0.60 in increments of $2 \%$ of the equilibrium volume $V_{0}$. After each reduction in the volume, the unit cell shape, as well as the atomic

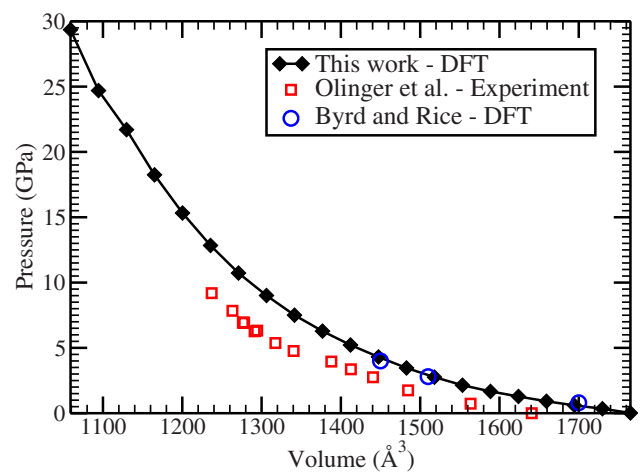

FIG. 2. (Color online) The isothermal EOS compared with the experiment (Ref. 3) and other DFT calculations (Ref. 22). The range in volume shown is $V / V_{0}=1.00-0.60$.

coordinates, was relaxed under the constraint of constant volume. The same tolerances in energy and forces were used as in the relaxation of the experimental structure.

For the simulation of uniaxial compression, the unit cell was rotated such that the desired compression direction was oriented along the positive $x$-axis (Fig. 1). Then, the $x$-components of each lattice vector were scaled in steps of $2 \%$ of $V_{0}$ from $V / V_{0}=1.00$ to 0.70 . The directions for compression were $\langle 100\rangle,\langle 010\rangle,\langle 001\rangle,\langle 110\rangle,\langle 101\rangle,\langle 011\rangle$, and $\langle 111\rangle$. Note that $\langle h k l\rangle$ is used to specify the direction that is perpendicular to the Miller plane $(h k l)$ as used by Dick et $a l^{31}$

At higher compression, it became difficult and costly to reduce the maximum force on any atom below $0.03 \mathrm{eV} / \AA$. Hence, the results for uniaxial compression have a maximum force of $0.03 \mathrm{eV} / \AA$ for $V / V_{0}$ greater than 0.80 , but the maximum force is about $0.05 \mathrm{eV} / \AA$ or less for all structures with $V / V_{0}=0.80-0.70$.

\section{EQUILIBRIUM PROPERTIES AND HYDROSTATIC EOS}

The calculated lattice parameters and unit-cell volume of $\alpha$-RDX at zero pressure are compared with roomtemperature experimental data ${ }^{2}$ in Table I. The percent difference in lattice parameters between our calculations and experiment ranges from $1.6 \%$ for $b$ to $3.7 \%$ for $c$. The agreement for $\alpha$-RDX is similar to our previous work for PETN (Ref. 23) and $\mathrm{HMX}^{24}$ Further, the lattice parameters and unit-cell volume are overestimated, which is a typical result of the PBE generalized gradient approximation functional. It has been suggested that the poor description of van der Waals interactions in DFT functionals is responsible for the overestimation of the unit-cell size for molecular crystals. ${ }^{21}$

The isothermal EOS is shown in Fig. 2 along with the

TABLE I. Calculated lattice constants of $\alpha$-RDX at zero pressure.

\begin{tabular}{lcccc}
\hline \hline & $a$ & $b$ & $c$ & \\
Work & $(\AA)$ & $(\AA)$ & $(\AA)$ & $\left(\AA^{3}\right)$ \\
\hline Choi and Prince $^{\mathrm{a}}$ & 13.182 & 11.574 & 10.709 & 1633.9 \\
This work & $13.522(+2.6 \%)$ & $11.758(+1.6 \%)$ & $11.102(+3.7 \%)$ & $1765.16(+8.0 \%)$ \\
\hline \hline Reference 2. & & &
\end{tabular}




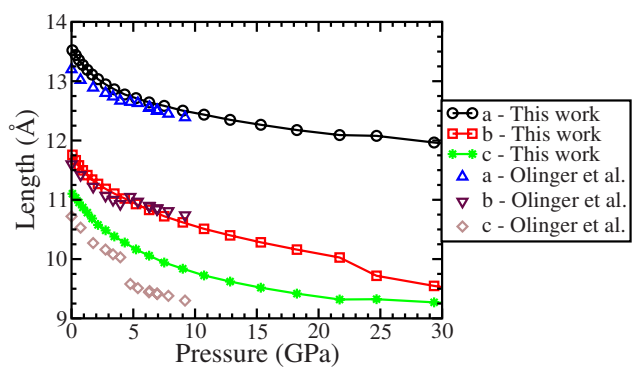

FIG. 3. (Color online) The lattice constants of $\alpha$-RDX as a function of pressure from hydrostatic compression calculations. The maximum pressure shown corresponds to $V / V_{0}=0.60$.

experimental data of Olinger et al. $^{3}$ The results from hydrostatic compression of Yoo et al. ${ }^{4}$ are not included because the volume at ambient conditions $V_{0}$ was not reported. However, both experiments ${ }^{3,4}$ agreed with each other as far as pressure versus volume dependence is concerned. According to the experimental results ${ }^{3}$ shown in Fig. 2, a phase transition to $\gamma$-RDX occurs between 3.95 and $4.76 \mathrm{GPa}$. There is a change in the curvature of the experimental isotherm near this interval of pressure. In our calculations, this phase transition was not observed. This is because these two phases are separated by an energy barrier, and the static relaxation technique cannot simulate the transition from one local minimum to another. The simulation of a phase transition requires the application of special transition-state search techniques, and this will be the subject of future research. In contrast to our previous results on PETN (Ref. 23) and HMX, ${ }^{24}$ there is a substantial deviation of the theoretical EOS from experiment. A similar problem has been encountered in previous calculations of hydrostatic compression of RDX by Byrd and Rice, ${ }^{22}$ and this disagreement is attributed to poor description of van der Waals interactions by DFT.

The lattice parameters as a function of pressure are shown in Fig. 3. The experimental hydrostatic-compression data of Olinger et $a l^{3}$ are shown for comparison. The agreement between experiment and our calculations of $a$ and $b$ lattice constants increases with pressure from 0 to $4 \mathrm{GPa}$, where the phase transition occurs in experiment.

The bulk modulus and its pressure derivative were found by fitting the calculated hydrostatic-compression data to three common fitting forms for pressures below 4.3 GPa. The third-order Birch-Murnaghan (BM) EOS

$$
P=\frac{3}{2} B_{0}\left(x^{-7}-x^{-5}\right)\left[1+\frac{3}{4}\left(B_{0}^{\prime}-4\right)\left(x^{-2}-1\right)\right]
$$

where $x=\left(V / V_{0}\right)^{1 / 3}$, is commonly used for energetic materials and was included in our fitting forms. Another form claimed to be accurate for a broad range of materials ${ }^{32}$ is the Vinet (V) EOS

$$
P=\frac{B_{0}[3(1-x)] e^{(x-1)\left(V_{0}^{1 / 3} / l_{v}\right)}}{x^{2}}
$$

where the $\mathrm{V}$ scaling length $l_{v}$ is given by

$$
l_{v}=\frac{2 V_{0}^{1 / 3}}{3\left(B_{0}^{\prime}-1\right)} \text {. }
$$

The final form, which was used in the analysis by Olinger et al., ${ }^{3}$ is from the shock Hugoniot conservation (SHC) equations

$$
\begin{aligned}
& U_{s}=\left[P V_{0} /\left(1-V / V_{0}\right)\right]^{1 / 2}, \\
& U_{p}=\left[P V_{0}\left(1-V / V_{0}\right)\right]^{1 / 2},
\end{aligned}
$$

where the pseudoshock velocity $U_{s}$ and pseudoparticle velocity $U_{p}$ were fit to the line $U_{s}=c+s U_{p}$ to find the constants $c$ and $s$. From these constants, the bulk modulus $B=c^{2} / V_{0}$ and its pressure derivative $B_{0}^{\prime}=4 s-1$ were calculated. Table II shows the results of our calculations compared with the experiment. $^{3,4,8,10-12}$ Note that calculation of the bulk modulus via the methods of Voigt and Reuss requires the elastic constants of the material. ${ }^{33}$ The values reported for the bulk modulus from experiment range from 9.8 to $13.9 \mathrm{GPa}$, and our results are within this range for each of the three fitting forms above. The pressure derivative of the bulk modulus

\begin{tabular}{|c|c|c|c|c|c|c|c|c|}
\hline Work & $B_{\text {Voigt }}$ & $B_{\text {Reuss }}$ & $B_{0, \mathrm{BM}}$ & $B_{0, \mathrm{BM}}^{\prime}$ & $B_{0, \mathrm{~V}}$ & $B_{0, \mathrm{~V}}^{\prime}$ & $B_{0, \mathrm{SHC}}$ & $B_{0, \mathrm{SHC}}^{\prime}$ \\
\hline Haussuhl $^{\mathrm{a}}$ & 11.38 & 11.05 & $\cdots$ & $\cdots$ & $\cdots$ & $\cdots$ & $\cdots$ & $\cdots$ \\
\hline Schwarz et al. ${ }^{\mathrm{b}}$ & 11.94 & 11.67 & $\cdots$ & $\cdots$ & $\cdots$ & $\cdots$ & $\cdots$ & $\cdots$ \\
\hline Haycraft et al. ${ }^{\mathrm{c}}$ & 12.05 & 11.92 & $\cdots$ & $\cdots$ & $\cdots$ & $\cdots$ & $\cdots$ & $\cdots$ \\
\hline Olinger et $a l .{ }^{\mathrm{d}}$ & $\cdots$ & $\cdots$ & 12.1 & 8.7 & 12.3 & 8.2 & 13.0 & 6.6 \\
\hline Yoo et al. ${ }^{\mathrm{e}}$ & $\cdots$ & $\cdots$ & 13.9 & 5.8 & $\cdots$ & $\cdots$ & $\cdots$ & $\cdots$ \\
\hline Davidson $e t a l .{ }^{\mathrm{f}}$ & $\cdots$ & $\cdots$ & 9.8 & 11.4 & $\cdots$ & $\cdots$ & $\cdots$ & $\cdots$ \\
\hline This work & $\cdots$ & $\cdots$ & 10.1 & 8.4 & 10.3 & 7.8 & 12.3 & 5.0 \\
\hline
\end{tabular}
from this work, with the exception of the value from the SHC fit, is also consistent with the range of experimental values.

TABLE II. Bulk modulus and its pressure derivative compared with experimental values.

\footnotetext{
${ }^{\mathrm{a}}$ Reference 10.

${ }^{\mathrm{b}}$ Reference 12.

${ }^{\mathrm{c}}$ Reference 11.

${ }^{\mathrm{d}}$ Reference 3 .

${ }^{\mathrm{e}}$ Reference 4.

${ }^{\mathrm{f}}$ Reference 8 .
} 

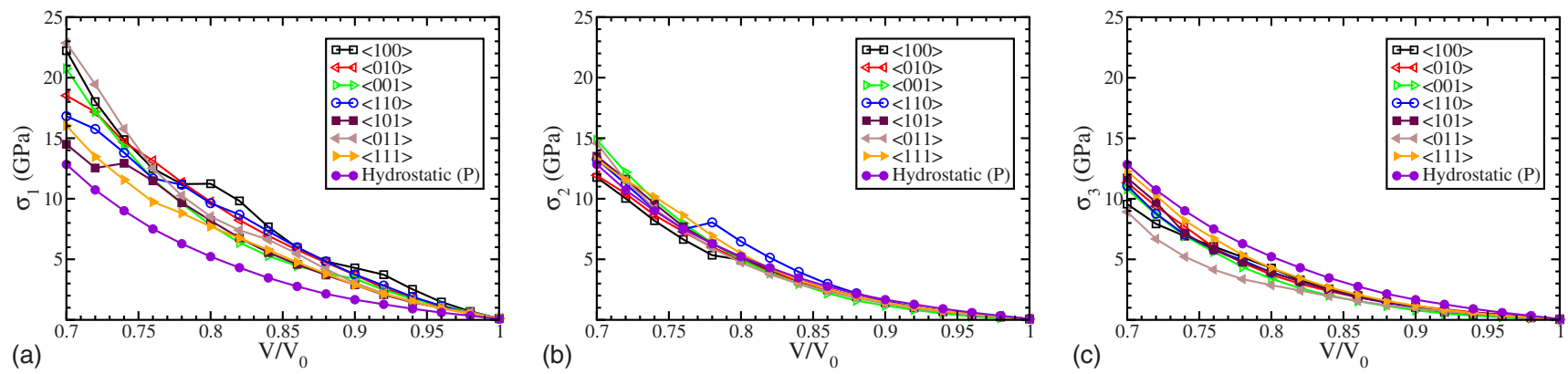

FIG. 4. (Color online) Principal stresses of $\alpha$-RDX under uniaxial compression. The principal stresses are arranged such that $\sigma_{1}$ is the greatest and $\sigma_{3}$ is the smallest. The pressure from the hydrostatic compression calculations is included for comparison.

\section{UNIAXIAL COMPRESSIONS}

The principal stresses of $\alpha$-RDX under uniaxial compression are shown in Fig. 4. Owing to the fact that the nondiagonal elements of the stress tensor acquire nonzero values upon uniaxial compression, it is necessary to transform the stress tensor to the principal axes to obtain the principal stresses, which are the eigenvalues of the stress tensor. The anisotropic behavior of the principal stresses in $\alpha$-RDX can clearly be observed from the greatest principal stress $\sigma_{1}$. Up to $V / V_{0}=0.80$, the $\langle 100\rangle$ compression shows the greatest value for $\sigma_{1}$, and the $\langle 001\rangle,\langle 101\rangle$, and $\langle 111\rangle$ directions have the smallest values of $\sigma_{1}$. However, some of the directions, including $\langle 100\rangle$ and $\langle 101\rangle$, exhibit nonmonotonic growth of $\sigma_{1}$ at higher compression.

The change in energy per atom under uniaxial compression is shown in Fig. 5. The greatest change in energy is observed in the $\langle 100\rangle$ compression as well, which is followed closely by the $\langle 010\rangle$ compression. As compression increases, the $\langle 001\rangle$ and $\langle 011\rangle$ energy curves begin to approach the $\langle 100\rangle$ and $\langle 010\rangle$ curves. The $\langle 111\rangle$ compression appears to have the slightest change in energy during compression, and all uniaxial compressions raise the energy more than hydrostatic compression due to the additional geometrical constraint imposed on the system.

The band gap under uniaxial compression is shown in Fig. 6. The $\langle 100\rangle$ compression shows a significantly greater reduction in the band gap relative to the other directions studied. It is noteworthy that the $\langle 011\rangle$ compression shows less of a reduction in the band gap than hydrostatic compres-

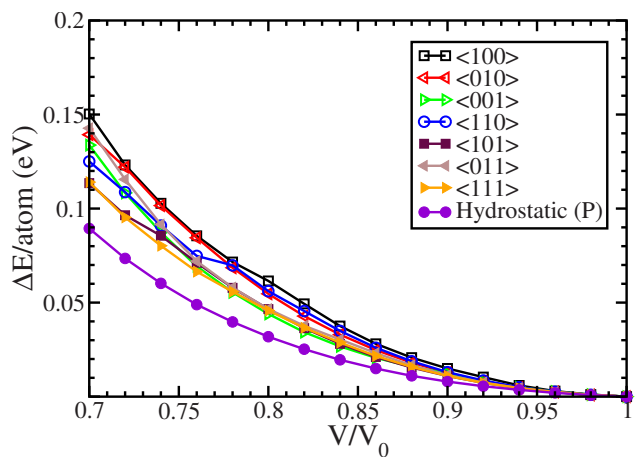

FIG. 5. (Color online) Change in energy per atom of $\alpha$-RDX under uniaxial compression. The change in energy per atom under simulated hydrostatic compression is included for comparison. sion for all shown values of $V / V_{0}$. This is also true for the $\langle 001\rangle$ compression at higher compression. Theoretical work has been done that suggests the band-gap reduction in energetic materials under compression might play a role in sensitivity to shock. ${ }^{18,19}$

The maximum shear stresses as a function of compression for all directions studied are shown in Fig. 7. The maximum shear stresses are calculated from the principal stresses by $\tau=\left(\sigma_{i}-\sigma_{j}\right) / 2$, where $i$ and $j(\neq i)$ take the values 1,2 , and 3 . The maximum shear stress $\tau_{\max }$ is found by using $i=1$ and $j=3$, but the values of $i$ and $j$ used to find $\tau_{\text {mid }}$ and $\tau_{\text {min }}$ depend on the principal stresses for the given compression direction.

We have labeled the greatest maximum shear stress as $\tau_{\max }$, the smallest as $\tau_{\min }$, and the intermediate value by $\tau_{\text {mid. }}$. Figure 7 indicates that the maximum shear stresses are anisotropic for $\alpha$-RDX. Despite the changes in curvature in most directions with compression, the $\langle 100\rangle$ and $\langle 011\rangle$ compressions consistently show greater values of $\tau_{\max }$ and $\tau_{\text {mid. }}$. Meanwhile, the $\langle 101\rangle$ and $\langle 111\rangle$ curves show consistently lower values for these shear stresses. Further, the $\langle 111\rangle$, $\langle 101\rangle,\langle 100\rangle$, and, to a lesser extent, $\langle 110\rangle$ curves exhibit noticeable nonmonotonic behavior of the shear stresses upon compression.

Our previous calculations for PETN indicated that the shear stresses were greater under uniaxial compression for directions with observed higher sensitivity and smaller for the relatively insensitive direction. Unlike PETN, experimental results regarding a possible anisotropy in sensitivity for RDX are not currently available. Therefore, it is not pos-

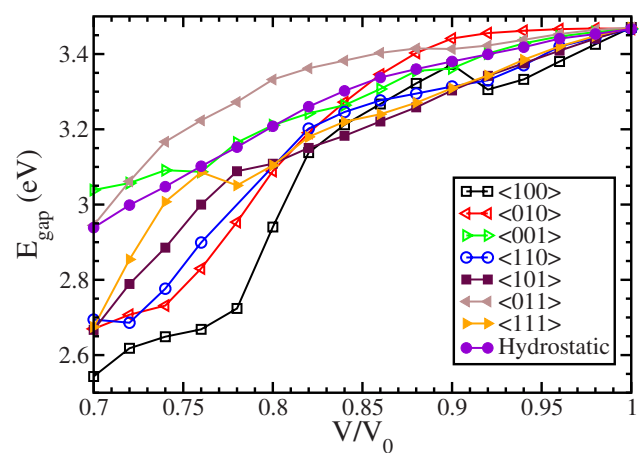

FIG. 6. (Color online) Band gap of $\alpha$-RDX under uniaxial compression. The band gap from simulated hydrostatic compression is included for comparison. 

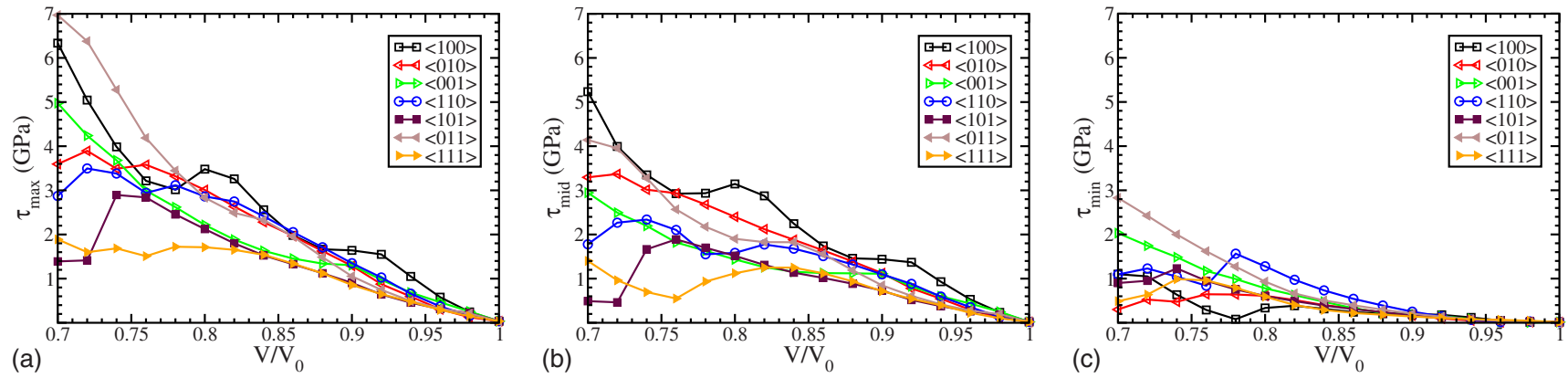

FIG. 7. (Color online) Maximum shear stresses $\tau_{\max }, \tau_{\text {mid }}$, and $\tau_{\min }$ under uniaxial compression.

sible at this time to support such a correlation based on our calculations until new experiments on shock sensitivity of RDX are performed.

\section{CONCLUSIONS}

We performed first-principles DFT calculations of both hydrostatic and uniaxial compressions of $\alpha$-RDX. The calculated structure at zero pressure had lattice parameters that agreed to within about $2 \%-4 \%$ of experiment. The $0 \mathrm{~K}$ isotherm was calculated and compared with the experiment. The directions studied for the uniaxial compression calculations were $\langle 100\rangle,\langle 010\rangle,\langle 001\rangle,\langle 110\rangle,\langle 101\rangle,\langle 011\rangle$, and $\langle 111\rangle$ within the Pbca space group. For each compression, the change in energy per atom, band gap, principal stresses, and maximum shear stresses as a function of reduced volume were calculated. The results indicate that each of these physical quantities shows a significant anisotropy.

\section{ACKNOWLEDGMENTS}

The work at USF was supported by the Office of Naval Research (ONR) through the Naval Research Laboratory (NRL) and partly by the Army Research Office (ARO) through the Multi-University Research Initiative (MURI) on Insensitive Munitions (IM) and the Center for Integrated Functional Materials at the USF Physics department. The work at Caltech was supported by ONR and the ARO through the MURI on IM. The work at NRL was supported by ONR both directly and through NRL. The computations were performed using NSF Teragrid computational facilities.

${ }^{1}$ P. J. Miller, S. Block, and G. J. Piermarini, Combust. Flame 83, 174 (1991).

${ }^{2}$ C. S. Choi and E. Prince, Acta Crystallogr., Sect. B: Struct. Crystallogr. Cryst. Chem. 28, 2857 (1972).

${ }^{3}$ B. Olinger, B. Roof, and H. Cady, Symposium on High Dynamic Pressures (Commissariat a l'Energie Atomique, Saclay, France, 1978), pp. 3-8.

${ }^{4}$ C.-S. Yoo, H. Cynn, W. M. Howard, and N. Holmes, in Proceedings of the Eleventh International Detonation Symposium, Snowmass Village, CO,
1998 (unpublished), p. 951.

${ }^{5}$ B. J. Baer, J. Oxley, and M. Nicol, High Press. Res. 2, 99 (1990).

${ }^{6}$ J. A. Ciezak, T. A. Jenkins, Z. Liu, and R. J. Hemley, J. Phys. Chem. A 111, 59 (2007).

${ }^{7}$ Z. A. Dreger and Y. M. Gupta, J. Phys. Chem. B 111, 3893 (2007).

${ }^{8}$ A. J. Davidson, I. D. H. Oswald, D. J. Francis, A. R. Lennie, W. G. Marshall, D. I. A. Millar, C. R. Pulham, J. E. Warren, and A. S. Cumming, Cryst. Eng. Comm. 10, 162 (2008).

${ }^{9}$ R. J. Karpowicz and T. B. Brill, J. Phys. Chem. 88, 348 (1984).

${ }^{10}$ S. Haussühl, Z. Kristallogr. 216, 335 (2001).

${ }^{11}$ J. J. Haycraft, L. L. Stevens, and C. J. Eckhardt, J. Chem. Phys. 124, 024712 (2006).

${ }^{12}$ R. B. Schwarz, D. E. Hooks, J. J. Dick, J. I. Archuleta, and A. R. Martinez, J. Appl. Phys. 98, 056106 (2005).

${ }^{13}$ D. E. Hooks, K. J. Ramos, and A. R. Martinez, J. Appl. Phys. 100, 024908 (2006).

${ }^{14}$ D. E. Hooks, K. J. Ramos, and D. F. Bahr, in Shock Compression of Condensed Matter, edited by M. Elert, M. D. Furnish, R. Chau, N. Holmes, and J. Nguyen (AIP, Melville, 2007), p. 789.

${ }^{15}$ A. B. Kunz, Phys. Rev. B 53, 9733 (1996).

${ }^{16}$ M. M. Kuklja and A. B. Kunz, J. Phys. Chem. Solids 61, 35 (2000).

${ }^{17}$ M. M. Kuklja and A. B. Kunz, J. Appl. Phys. 87, 2215 (2000).

${ }^{18}$ M. M. Kuklja, E. V. Stefanovich, and A. B. Kunz, J. Chem. Phys. 112, 3417 (2000)

${ }^{19}$ M. M. Kuklja, B. P. Aduev, E. D. Aluker, V. I. Krasheninin, A. G. Krechetov, and A. Y. Mitrofanov, J. Appl. Phys. 89, 4156 (2001).

${ }^{20}$ T. D. Sewell and C. M. Bennett, J. Appl. Phys. 88, 88 (2000).

${ }^{21}$ E. F. C. Byrd, G. E. Scuseria, and C. F. Chabalowski, J. Phys. Chem. B 108, 13100 (2004).

${ }^{22}$ E. F. C. Byrd and B. M. Rice, J. Phys. Chem. C 111, 2787 (2007).

${ }^{23}$ M. W. Conroy, I. I. Oleynik, S. V. Zybin, and C. T. White, Phys. Rev. B 77, 094107 (2008).

${ }^{24}$ M. W. Conroy, I. I. Oleynik, S. V. Zybin, and C. T. White, J. App. Phys. 104, 053506 (2008).

${ }^{25}$ G. Kresse and J. Furthmuller, Phys. Rev. B 54, 11169 (1996).

${ }^{26}$ G. Kresse and J. Furthmuller, Comput. Mater. Sci. 6, 15 (1996).

${ }^{27}$ J. P. Perdew, K. Burke, and M. Ernzerhof, Phys. Rev. Lett. 77, 3865 (1996).

${ }^{28}$ J. P. Perdew, K. Burke, and M. Ernzerhof, Phys. Rev. Lett. 78, 1396 (1997).

${ }^{29}$ P. E. Blochl, Phys. Rev. B 50, 17953 (1994).

${ }^{30}$ G. Kresse and D. Joubert, Phys. Rev. B 59, 1758 (1999).

${ }^{31}$ J. J. Dick, R. N. Mulford, W. J. Spencer, D. R. Pettit, E. Garcia, and D. C. Shaw, J. Appl. Phys. 70, 3572 (1991).

${ }^{32}$ P. Vinet, J. Ferrante, J. R. Smith, and J. H. Rose, J. Phys. C 19, L467 (1986).

${ }^{33}$ R. Hill, Proc. Phys. Soc., London, Sect. A 65, 349 (1952). 